\title{
El papel del alumnado en la propuesta y diseño de acciones docentes
}

The role of students in the proposal and design of teaching activities

\author{
Fernando Castelló-Sirvent \\ ESIC Business and Marketing School (Campus Valencia) \\ fernando.castello@esic.edu \\ Vanessa Roger Monzó \\ ESIC Business and Marketing School (Campus Valencia) \\ vanessa.roger@esic.edu
}

Fecha presentación: 02/05/2017 | Aceptación: 16/11/2017 |Publicación: 22/06/2018

\begin{abstract}
Resumen
En esta investigación se presenta el Proyecto de Innovación Docente ComunicArte16, llevado a cabo durante el curso 2015-2016 en la asignatura Estructura Económica del Sector de la Comunicación del grado en Comunicación y Relaciones Públicas de ESIC Business \& Marketing School, en el que participaron 21 alumnos. ComunicArte16 tiene como objetivo incrementar la participación del alumnado a través de la propuesta y diseño de sus propias acciones docentes a realizar en el aula. A partir de las 14 acciones propuestas, se solicitó a cinco docentes especialistas en la materia que revelaran sus preferencias de acuerdo a los objetivos del currículo de la asignatura. Este proceso se ha articulado sobre cuatro ejes: analizar, conectar, experimentar y crear (ACEC), siguiendo como referencia el Modelo de Estilos de Aprendizaje de Felder-Silverman (FSLSM). Para la selección de acciones docentes de ComunicArte16 se siguió el Proceso Analítico Jerárquico (AHP) que, a través de la comparación pareada de alternativas de tipo autoexcluyente, brindó una prelación ponderada y dio lugar a la selección efectiva de 4 acciones, una para cada eje. El análisis de los resultados avala una alta aceptación de ComunicArte16 entre el alumnado, incrementando la participación activa en el aula y mejorando su compromiso a través de una mayor tasa de asistencia a clase.
\end{abstract}

Palabras clave: innovación educativa; EEES; experiencias de aprendizaje; Proceso Analítico Jerárquico (AHP)

\section{Resum}

En aquesta investigació es presenta el Projecte d'Innovació Docent ComunicArte16 dut a terme en el curs 2015-2016 en l'assignatura Estructura Econòmica del Sector de la Comunicació del grau en Comunicació i Relacions Públiques d'ESIC Business \& Marketing School, on van participar 21 alumnes. ComunicArte16 té com a objectiu incrementar la participació de l'alumnat a través de la proposta i disseny de les seues pròpies accions docents a realitzar a l'aula. A partir de les 14 accions proposades, es va sol-licitar a cinc docents especialistes en la matèria que revelaren les seues preferències d'acord amb els objectius del currículum de l'assignatura. Aquest procés s'ha articulat sobre quatre eixos: analitzar, connectar, experimentar i crear (ACEC), seguint com a referència el Model d'Estils d'Aprenentatge de FelderSilverman (FSLSM). Per a la selecció d'accions docents de ComunicArte16 es va seguir el Procés Analític Jeràrquic (AHP) que, a través de la comparació aparellada d'alternatives de tipus autoexcloents, va brindar una prelació ponderada i va donar lloc a la selecció efectiva de 4 accions, una per a cada eix. L'anàlisi dels resultats avala una alta acceptació de ComunicArte16 entre l'alumnat, incrementant la participació activa a l'aula i millorant el seu compromís a través d'una major taxa d'assistència a classe.

Paraules clau: innovació educativa; EEES; experiència d'aprenentatge; Procés Analític Jeràrquic (AHP)

\section{Abstract}

The ComunicArte16 Teaching Innovation Project is presented in this paper, in which 21 students participated. It was carried out in the academic course 2015-2016 in the subject Economical Structure of the Communication Sector of the degree in Communication and Public Relations of ESIC Business \& Marketing School.

ComunicArte16 aims to increase student participation through the proposal and design of their own teaching actions to be carried out in the classroom. From the 14 proposed actions, five teachers who are specialized in the subject were 
asked to reveal their preferences according to the objectives of the subject's curriculum. This process has been articulated on four axes: analyze, connect, experiment and create (ACEC), following the Felder-Silverman Learning Styles Model (FSLSM). For the selection of teaching actions of ComunicArte16 the Hierarchical Analytical Process (AHP) was followed and which, through the paired comparison of alternatives of self-excluding type, provided a weighted priority and resulted in the effective selection of 4 actions, one for each axis. The analysis of the results supports a high acceptance of ComunicArte16 among the students, increasing active participation in the classroom and improving their commitment through a higher attendance rate.

Key Words: educational innovation; EHEA; learning experience; Analytic Hierarchy Process (AHP)

\section{Introducción y objetivos}

Un aspecto fundamental sobre el que se instaura el Espacio Europeo de Educación Superior (EEES) es el de mejorar la calidad de la enseñanza universitaria, centrándose en los procesos formativos y, especialmente, en los resultados de aprendizaje articulados en torno a competencias transversales y específicas. Resulta crucial desarrollar estrategias docentes que favorezcan el aprendizaje autónomo y continuo de los estudiantes. La mera transmisión de conocimientos queda sustituida por un esquema que fomenta la adquisición de nuevas habilidades, capacidades y aptitudes a través del autoaprendizaje (Martín et al. 2014). Las acciones llevadas a cabo por el profesorado abarcan, más allá de las tradicionales clases magistrales y sus correspondientes prácticas de laboratorio, un amplio abanico de posibilidades (Cabero et al., 2006). Esta circunstancia, unida a la generalización del trabajo colaborativo como metodología docente en las universidades españolas (Vallet et al., 2017) y la integración de las tecnologías de la información y comunicación (TIC) están modificando sustancialmente el ámbito de la enseñanza universitaria (Cabero y Marín, 2014).

A partir de estas premisas, los objetivos de este estudio son:

1. Presentar el Proyecto de Innovación Docente ComunicArte16, dirigido a incrementar la participación activa del alumnado, que tiene la posibilidad de proponer y diseñar las posibles acciones docentes a realizar en el aula.

2. Proponer un modelo vertebrado desde cuatro ejes: analizar, conectar, experimentar y crear (ACEC), siguiendo como referencia el Modelo de Estilos de Aprendizaje de Felder-Silverman (FSLSM) para implementar ComunicArte16.

3. Evaluar el grado de aceptación de ComunicArte16, analizando la participación y el compromiso del alumnado.

En primer lugar, se ha realizado una revisión de la literatura a partir de la cual se propone la secuencia ACEC; a continuación, se describe ComunicArte16 y su metodología específica y, por último, se analizan los resultados obtenidos.

\section{Marco teórico}

El Documento Marco sobre la Integración del Sistema Universitario Español en el EEES establece que las instituciones de educación superior deben tener "una orientación profesional, es decir, deberán proporcionar una formación universitaria en la que se integren armónicamente las competencias genéricas básicas, las competencias transversales relacionadas con la formación integral de las personas y las competencias más específicas que posibiliten una orientación profesional que permita a los titulados una integración en el mercado de trabajo" (Ministerio de Educación, Cultura y Deportes (2003: 7). Por tanto, y teniendo en cuenta la evolución en las necesidades formativas que demanda la sociedad, es indispensable promover experiencias innovadoras en los procesos de enseñanzaaprendizaje con el fin de capacitar al alumno en las destrezas que desempeñará durante su vida profesional, dado que el mercado laboral es muy cambiante y competitivo (Roger-Monzó et al. 2015). No en vano, la conceptualización de competencias se encuentra unida a la acción, a la experiencia y al contexto socioprofesional (Tejada, 2012).

Desde hace algunos años, en la comunidad educativa se está reivindicando la participación activa y real de los estudiantes. Son muchas las voces que se expresan en esta dirección (Martínez de Miguel, 2007). Por tanto, las estrategias docentes en el ámbito de la enseñanza superior deben centrarse "en una concepción del aprendizaje constructivista, en el que el estudiante es eje central en la creación de significado y el docente mediador entre el conocimiento y el alumnado" (Olmedo, 2013: 41). En este sentido, se han desarrollado numerosas metodologías docentes basadas en la investigación y la experimentación, vinculadas a realidades sociales de los estudiantes para que se sientan identificados con su entorno (Porlán, 1993; García Pérez, 2000).

Felder y Silverman $(1988,2002)$ establecen un Modelo de Estilos de Aprendizaje (FSLSM) centrado en el alumnado del ámbito de educación universitaria, y que queda estructurado en 4 dimensiones ${ }^{1}$ que toman 2 estilos opuestos por cada dimensión: percepción (sensitivo-intuitiva), entrada (visual-verbal), procesamiento (activo-reflexiva) y comprensión (secuencial-global).

En este sentido, los estudiantes muestran diferentes preferencias respecto a su proceso de aprendizaje. Algunos pueden comprender conceptos rápidamente mediante las imágenes mientras que otros prefieren los textos y lecturas. Del mismo modo, existen alumnos que pueden manejar su desempeño en el dominio de las teorías mientras que otros aprenden a través de la experimentación y ejemplificación (Troung, 2016)

Desde su publicación inicial, el FSLSM se ha convertido en una referencia, habiendo sido replicado, adaptado y ampliado en múltiples ocasiones y en diversos contextos, tal y como se muestra a continuación.

\footnotetext{
1 Inicialmente, dicho modelo contemplaba 5 dimensiones con sus correspondientes estilos opuestos. Sin embargo, una revisión posterior realizada en 2002 sobre el estudio original, actualizó parcialmente el modelo, eliminando uno de los constructos. Esta es la razón por la que cuando se hace referencia a esta publicación se empleen las dos fechas (1988, 2002).
} 


\begin{tabular}{|l|l|l|l|}
\hline Año & Autores & Ámbito del estudio & Contexto \\
\hline 2017 & Joseph y Abraham & $\begin{array}{l}\text { Análisis del sistema de gestión de } \\
\text { aprendizaje a partid de las } \\
\text { herramientas que optimizan el } \\
\text { FSLSM }\end{array}$ & $\begin{array}{l}\text { E-learning } \\
\text { (Moodle) }\end{array}$ \\
\hline 2016 & Chang et al. & $\begin{array}{l}58 \text { estudiantes de la Southern Taiwan } \\
\text { University of Science and Technology }\end{array}$ & Web Mashup \\
\hline 2016 & Qodad et al. & $\begin{array}{l}\text { Propuesta de modelo que guie el } \\
\text { aprendizaje del alumnado al objeto } \\
\text { de mejorar su satisfacción }\end{array}$ & E-learning \\
\hline 2016 & $\begin{array}{l}\text { Muruganandam y } \\
\text { Srinivasan }\end{array}$ & $\begin{array}{l}\text { Análisis de aceptación de ajuste e- } \\
\text { learning sobre diferentes estilos en el } \\
\text { FSLSM a partir de dos muestras }\end{array}$ & E-learning \\
\hline 2015 & Jingyun y Takaiko & $\begin{array}{l}\text { 198 estudiantes de 4 universidades } \\
\text { chinas }\end{array}$ & Idiomas \\
\hline 2014 & Truong & $\begin{array}{l}\text { Revisión de la literatura sobre teorias } \\
\text { de estilos de aprendizaje en el } \\
\text { entorno de e-learning, }\end{array}$ & E-learning \\
\hline 2014 & Fu y Li & $\begin{array}{l}\text { 2.936 estudiantes de Shaanxi Normal } \\
\text { University, South China Normal } \\
\text { University y Qinghai Normal } \\
\text { University (China) }\end{array}$ & E-learning \\
\hline 2013 & Ventura et al. & $\begin{array}{l}\text { 269 estudiantes de Ingenieria de la } \\
\text { Universidad Nacional de Rosario } \\
\text { (Argentina) }\end{array}$ & FSLSM / ILS \\
\hline $\begin{array}{l}\text { 81 estudiantes de 2 escuelas } \\
\text { secundarias de Trabzon (Turquia) }\end{array}$ & E-learning \\
\hline & Ozyurt, et al. &
\end{tabular}

Tabla 1. Trabajos recientes de mayor relevancia a partir del FSLSM. Fuente: Elaboración propia

Cabe destacar que el trabajo de Özyurt et al. (2013) propone un sistema experto de e-learning (UZWEBMAT) desarrollado a partir de los estilos de aprendizaje tomados desde el FSLSM, mediante el diseño de un entorno de aprendizaje electrónico personalizado e inteligente. Los autores evalúan sus efectos sobre el aprendizaje de 81 estudiantes de $10^{\circ}$ grado de dos escuelas secundarias en Trabzon, Turquía. Por su parte, Fu y Li (2014) llevan a cabo el análisis de la relación existente entre la ansiedad en los sistemas de e-learning y el sentido de orientación espacial mostrado en grupos de estudiantes con diferentes estilos de aprendizaje.

Troung (2015) realiza una revisión de 51 trabajos predictores de estilos de aprendizaje en línea con clasificación automática, al objeto de lograr una optimización one-to-one del proceso de aprendizaje con una detección temprana de los estilos preferidos por los alumnos mediante el uso de algoritmos. De hecho, Joseph y Abraham (2017) analizan la capacidad instrumental de los diferentes tipos de estímulo a través del entorno Moodle y el modo en que logran impactar de forma más adecuada sobre los diferentes estilos de aprendizaje propuestos en el FSLSM.

Ventura et al. (2014) identifican los estilos de aprendizaje en estudiantes de ingeniería civil y electrónica, haciéndolo en entornos didácticos adaptados a las preferencias predominantes del alumnado de primer curso tras su ingreso a la universidad, y proponiendo una combinación de herramientas capaces de potenciar la coexistencia combinada y recurrente de diferentes estilos de aprendizaje en el alumnado analizado.

Por su parte, Qodad et al. (2016) proponen un modelo que permite guiar el aprendizaje del alumnado al objeto de mejorar su satisfacción. Estos autores lo hacen a partir de cinco modelos: dominio, aprendizaje, orientación al trabajo, instrucción y adaptabilidad. EI dominio se aborda desde los estándares SCORM; el aprendizaje a través del FSLSM; la orientación al trabajo fija el perfil deseado por la empresa contratante; la instrucción articula usos pedagógicos diferenciados que permiten la correcta asignación del objeto de aprendizaje; la adaptabilidad garantiza el encaje de las habilidades requeridas por el trabajo y las propias características del alumnado.

Como hemos indicado respecto a entornos virtuales de aprendizaje (Joseph y Abraham, 2017), el uso de las TIC resulta predominante en los diferentes análisis de adaptación del FSLSM. Así, Muruganandam y Srinivasan (2016) utilizan el lenguaje JAVA, y Chang et al. (2016) vinculan los estilos y actuaciones de aprendizaje dentro de un sistema de apoyo en el área de la enseñanza de idiomas, y analizan el grado de adaptabilidad e integración del FSLSM por medio de las tecnologías Mashup de base web a partir del diseño de un experimento que se llevó a cabo durante 8 semanas en la Southern Taiwan University of Science and Technology. Siguiendo en el contexto de las enseñanzas de idiomas, Jingyun y Takaiko (2015) relacionan los estilos FSLSM y las actuaciones de aprendizaje del alumnado a través de un sistema de apoyo que aplican sobre 198 estudiantes en 4 universidades chinas, combinando tanto estudiantes de ingeniería (60) como de lenguas (138).

En el FSLSM, el alumnado muestra su percepción a partir de una escala de preferencias respecto a los diferentes estilos de aprendizaje en un enfoque dicotómico, quedando el estilo resultante calificado en tres categorías: fuerte, moderado y equilibrado, en función de la intensidad de la polarización mostrada a nivel individual en cada dimensión. Aquellos individuos con estilos de polarización fuerte pueden llegar a presentar dificultades de aprendizaje si en el aula no se encuentran actividades docentes orientadas a su extremo o si el ambiente no es dirigido por el docente en la dirección en que se muestra fuerte (Ramírez y Rosas, 2014).

Por otro lado, la satisfacción del alumnado ha mostrado una fuerte vinculación con el rendimiento académico (Vélez y Roa, 2005). Asimismo, Castaño et al. (2015) evalúan el rendimiento académico a través de evidencias de aprendizaje, estableciendo fuerte vinculación entre rendimiento académico y la satisfacción del alumnado. Danielsen et al. (2009) revelan que la satisfacción con la escuela es una variable determinante en el progreso del estudiante que influye en el rendimiento escolar. De igual modo, Paulsen y Feldman (2005) han demostrado que la satisfacción de un estudiante predice su rendimiento académico. Además, la figura del docente adquiere un papel importante en la satisfacción del estudiante y en su aprendizaje (Baños et al., 2017).

Conocer los estilos de aprendizaje del alumnado facilita que el profesorado pueda modular los diferentes estilos de enseñanza disponibles. Por ello, se han considerado los diferentes estilos de enseñanza, siguiendo a González-Peiteado y Pino-Juste (2013) que se distribuyen en: reflexivo, cooperador, académico, individual, innovador e indagador.

Al objeto de lograr un adecuado ajuste entre los estilos de enseñanza (González-Peiteado y Pino-Juste, 2013) y de aprendizaje (Felder y Silverman, 1988, 2002) y tomando en consideración el Documento Marco del EEES que determina la necesidad de promover una formación que favorezca la futura integración del alumnado en el ámbito profesional, se plantea un modelo integrador alineado con los estudios anteriores. Se trata del Modelo ACEC, que se imbrica con las demandas del contexto educativo actual y se estructura en cuatro ejes: analizar, conectar, experimentar y crear, tomando en consideración la combinación de estilos de enseñanza-aprendizaje empleados de manera alternada y articulada para mejorar el desempeño en la práctica profesional (Ventura et al., 2014).

Los cuatro ejes sobre los que se articula el Modelo ACEC requieren que el profesorado responsable cuente con una amplia variedad de habilidades docentes al tiempo que emplea metodologías plenamente coherentes con 
una concepción centrada en el aprendizaje (Ballesta et al., 2011), y debe moverse dentro de un continuum, combinando rasgos diferenciales que mejoren el ajuste con las preferencias del alumnado (González-Peiteado y Aznar-Cuadrado, 2010).

La propuesta concede especial importancia al aprendizaje cooperativo puesto que, en comparación con otros métodos convencionales, posee efectos positivos sobre el rendimiento académico (Goikoetxea y Pascual, 2005). Cabe subrayar que la incorporación de los entornos virtuales de aprendizaje como Moodle favorece de manera notable el trabajo cooperativo entre estudiantes, dado que comparten, trabajan y aprenden en un espacio virtual, logrando así los objetivos que se plantean (Gómez-Camarero et al., 2010). En este sentido, Domingo y Fuentes (2010: 171) consideran que es "esencial desarrollar una innovación pedagógica en profundidad, aprovechando las TIC".

Del mismo modo, la integración de las redes sociales en la enseñanza implica que el proceso de aprendizaje de los estudiantes posea un enfoque colaborador (Barajas y Álvarez, 2013; Bernal y Angulo, 2013). Cabero y Marín (2014) establecen que las redes sociales ofrecen un componente social e interactivo que favorece una educación creativa, participativa e interactiva, permitiendo interacción y trabajo conjunto entre profesores y alumnos (Olmos-Migueláñez et al., 2014). El aprendizaje con redes sociales incrementa el interés por la formación, así como el grado de motivación de los alumnos, ya que tienen la posibilidad de interactuar con otros estudiantes (Torregrosa, 2010).

No obstante, Guitert y Pérez (2013) señalan la necesidad de concentrarse en el propio proceso de aprendizaje, más allá de las herramientas utilizadas o del contexto en el cual tiene lugar. En numerosos países, los procesos de innovación educativa se han centrado en la "incorporación eficaz de las TIC en un sistema educativo de estructuras anacrónicas" (Arancibia-Herrera et al., 2014, p. 76).

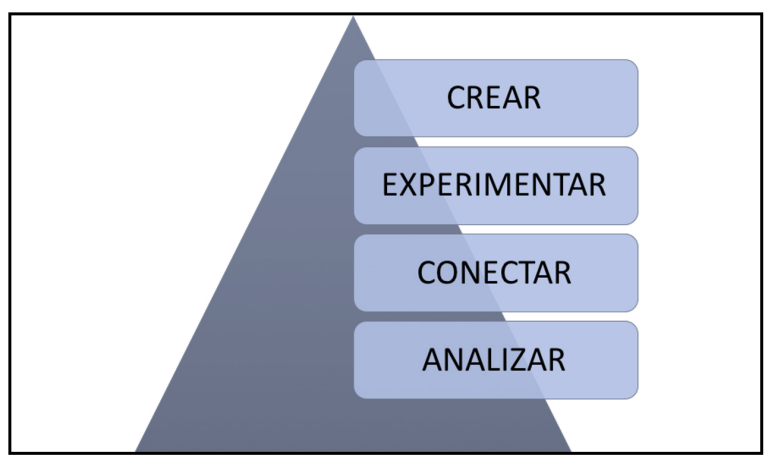

Figura 1. Ejes ACEC. Fuente: Elaboración propia

El enfoque secuencial de ACEC se vertebra a partir de un conjunto de competencias innovadoras-emprendedoras que deben facilitar, en última instancia, la integración profesional futura de los alumnos en el mercado laboral. A continuación, se describen los diferentes ejes ACEC.

\section{Analizar}

El primer eje pasa por desarrollar las competencias de análisis del alumno centrando el ámbito de acción sobre aquellas experiencias docentes que permitan el desarrollo de habilidades prospectivas siguiendo el enfoque aportado por González-Peiteado y Pino-Juste (2013). En última instancia, debe lograr situar al alumno en una posición que le permita articular iniciativas proactivas en torno a la formulación futura del marco referencial que le es propio en atención a su área de conocimientos.

\section{Conectar}

El alumno debe lograr conectar con la práctica profesional que se lleva a cabo en el presente, pero tomando conciencia de las iniciativas que se sitúan en la vanguardia de innovación tecnológica u organizativa. Las acciones docentes deben lograr que el alumno asimile la caracterización y dinámica competitiva del sector empresarial en el que se desempeñan sus salidas profesionales.

Las experiencias docentes realizadas en esta etapa deben lograr una observación reflexiva del entorno profesional y de la realidad social que es susceptible de vivenciar el alumno (García Pérez, 2000), de tal modo que pueda identificar el presente y anticipar el futuro de su sector. En definitiva, el alumnado debe desarrollar competencias vinculadas al autoaprendizaje, desde la acción y en un contexto de convivencia grupal (Monereo y Pozo, 2003).

\section{Experimentar}

En esta etapa las actividades de aula deben reforzar las habilidades emprendedoras de los alumnos, fomentando la experimentación de las herramientas y nuevas metodologías que se sitúan en la vanguardia profesional. La interacción del alumno con un marco teórico previamente asimilado es primordial para que éste pueda interiorizar las posibilidades competitivas disponibles.

Esta etapa devuelve al alumno la comprensión del modo en que se genera su propio conocimiento, situándole en el centro del proceso de aprendizaje como un importante actor proactivo y no como su destinatario pasivo (Piscitelli et al., 2010).

Cabe lograr desde la práctica (conocimiento tácito) la iteración reflexiva de todo el cuerpo teórico con que se presenta el currículo del alumno (conocimiento expreso), cristalizado a través de un nivel de comprensión más profundo de los mecanismos que dan lugar a este proceso de aprendizaje basado en la experiencia (Nonaka y Takeuchi, 1995).

\section{Crear}

En esta etapa no se pretende tanto configurar experiencias docentes dirigidas a fomentar la creatividad como favorecer capacidades propias de rutinas innovadoras y de tipo disruptivo, haciendo posible que los alumnos consoliden la fase de experimentación anterior tras eclosionar como un potente instrumento de creación de propuestas de valor que resulten plenamente competitivas en el mercado.

En este sentido, el trabajo colaborativo y el impulso de la experimentación de las metodologías didácticas con soporte TIC (Domingo y Fuentes, 2010; González, 2016), utilizados de forma transversal en las enseñanzas de ámbito universitario para la generación de ideas y nuevas propuestas, resultan centrales para despertar en los alumnos el interés sobre la filosofía edupunk ("Do it yourself"), como sugiere el Proyecto Facebook (Piscitelli et al., 2010).

De este modo, las metodologías de aprendizaje deben facilitar competencias para realizar la síntesis de información consolidada en forma de conocimiento experiencial susceptible de permitir su aplicación sobre problemas reales. De hecho, el conocimiento reside en la 
acción y surge de una ejecución espontánea y hábil (Tejada, 2012).

EI Modelo ACEC invita al alumnado a interiorizar el uso de las herramientas disponibles, incidiendo en sus competencias emprendedoras e innovadoras a partir de la conexión continuada con el marco teórico previamente asimilado, para finalmente ser capaz de crear propuestas de valor que, en su caso, pudieran resultar plenamente competitivas.

\section{Descripción del estudio y metodología \\ 3.1. Descripción del estudio}

El Proyecto de Innovación Docente ComunicArte16 se ha llevado a cabo durante el curso 2015-2016 en la asignatura Estructura Económica del Sector de la Comunicación, incluida en el grado en Comunicación y Relaciones Públicas de ESIC Business \& Marketing School (Campus Valencia), centro universitario adscrito a la Universidad Miguel Hernández de Elche. En el proyecto participaron un total de 19 alumnos.

ComunicArte16 aplica el Modelo ACEC, tomándolo como propuesta conceptual para este proyecto de innovación, y tiene como objetivo impactar positivamente sobre la participación activa y el compromiso del alumnado, ofreciéndole la posibilidad de diseñar sus propias acciones docentes.

El procedimiento fue el siguiente:

1. El alumnado participó activamente en el diseño de acciones docentes por medio de dinámicas grupales. Bajo la supervisión del profesorado, los alumnos elaboraron un total de 56 propuestas.

2. El profesorado responsable de ComunicArte16 llevó a cabo una primera selección atendiendo a criterios de posibilidad en función de los recursos de aula, tecnológicos y de tipo económico, resultando 14 propuestas (4 para analizar; 4 para conectar; 3 para experimentar; 3 para crear).

3. Se eligieron 4 acciones docentes, una para cada eje ACEC, aplicando un Proceso Analítico Jerárquico (AHP) con el apoyo de cinco expertos en la materia.

4. Se programaron las diferentes acciones docentes y tras su celebración se recogieron valoraciones cuantitativas sobre la aceptación de ComunicArte16 por parte del alumnado, constatando una mejora en su participación activa y compromiso con el proceso.

El sector de la comunicación viene experimentando una evolución radical. Dentro del grado en Comunicación y Relaciones Públicas, el currículo de la asignatura Estructura Económica del Sector de la Comunicación consta de 6 créditos ECTS y aborda la evolución estratégica y funcional de medios, agencias y anunciantes. Un importante punto de análisis pasa por la transformación que sufren los mensajes y usuarios a partir de la utilización sistemática de las redes sociales. Es por ello que el conjunto de actividades docentes tenía como fin accionar en el alumno una profunda reflexión sobre las nuevas tendencias comunicativas que está experimentando la sociedad en su conjunto, permitiéndole tomar conciencia de las implicaciones empresariales y sobre los modelos de negocio que ello conlleva.
La asignatura configura su guía docente ofreciendo alta importancia a la evaluación continua. En este sentido, un $30 \%$ de la calificación final viene dada por actividades de tipo grupal. La programación establece los contenidos y criterios de evaluación a aplicar en la asignatura. Sin embargo, en lo referido a la evaluación continua deja abierta la configuración de las acciones docentes a realizar en el aula.

ComunicArte16 hizo posible que el alumno abordara de forma experiencial la relevancia social que ello implica sobre la libertad individual de los usuarios, así como sobre la nueva configuración de los sistemas de poder.

\subsection{Metodología}

\subsubsection{Secuencia-guía implementada}

A continuación, se muestra el orden de acciones que se ha seguido para desarrollar el Proyecto de Innovación Docente ComunicArte16 (anexo 1).

\section{Presentación inicial}

En esta sesión se incidió con especial interés sobre los ejes ACEC y, particularmente, sobre los objetivos a alcanzar en cada una de ellos.

\section{Generación de Ideas}

Se llevó a cabo una dinámica de brainstorming, procedimiento de trabajo en grupo orientado a obtener un gran número de ideas sobre un tema concreto, facilitando la participación múltiple de los estudiantes (Osborn, 1963).

Se generaron cuatro sesiones paralelas, formando subgrupos de 3-4 alumnos que abordaron de manera rotatoria los cuatro ejes ACEC y propusieron ideas para experiencias docentes orientadas a la consecución de los objetivos pedagógicos específicos descritos en cada etapa. Dichas sesiones fueron supervisadas por los profesores responsables del proyecto y dieron lugar a un conjunto de ideas que se perfilaron a través de dinámicas grupales. En el proceso, se apreció una alta efectividad, mejorando la participación y creatividad grupal, tal y como constataron Felder y Brent (2001).

Posteriormente, los profesores responsables crearon en Moodle, la plataforma virtual de aprendizaje con que cuenta ESIC, y a través de la herramienta de foro, cuatro hilos de debate: uno para cada eje ACEC. Los alumnos dispusieron de 48 horas para llevar a cabo su participación individual durante horario no lectivo, generando 56 propuestas no coincidentes.

El objetivo de este estadio radicaba en hacer que la participación activa resultara múltiple y regresiva, permitiendo que las intervenciones generaran al menos una propuesta por cada alumno, para cada eje ACEC, y que cada alumno complementara, ampliara o matizara, al menos, una propuesta de uno de sus compañeros. De acuerdo con Gras y Cano (2005), se pudo observar que el estímulo del pensamiento crítico de los alumnos se produjo junto a una mejora de sus competencias de argumentación desde un entorno de trabajo que les permitía comunicar y colaborar grupalmente.

\section{Selección de alternativas}

En un primer paso, el profesorado vinculado al proyecto realizó una selección preliminar de las alternativas propuestas por los alumnos para cada eje ACEC, obedeciendo a criterios de posibilidad económicofinanciera y de adecuación formal con respecto a los objetivos curriculares planteados por la asignatura. Se descartaron diversas experiencias docentes en base a 
los recursos de aula que requerían, pues aun pudiendo resultar de gran interés curricular, no eran aplicables por limitaciones presupuestarias y/o funcionales.

Finalmente, se tomaron 14 acciones docentes diseñadas por los alumnos para ComunicArte16 a partir de los 4 ejes ACEC (anexo 2).

Posteriormente, con el concurso de un grupo de cinco expertos y docentes, especialistas todos en el sector de la comunicación, se aplicó la metodología AHP.

La aplicación de métodos de decisión multicriterio encuentra una alta complejidad subyacente (DeTombe, 2001) debido en gran medida a la alta influencia que este tipo de elección muestra ante factores subjetivos derivados de los juicios de valor que subyacen a la persona o personas que toman la decisión (Berumen y Llamazares, 2007), por cuanto no requieren información cuantitativa sobre cada una de las alternativas.

En este sentido, el Proceso Analítico Jerárquico (AHP) (Saaty, 1980; Saaty 1990) se presenta como una metodología que ofrece alta robustez matemática para la selección de alternativas en presencia de problemas complejos, dividiendo una decisión difícil en un conjunto de decisiones de mayor sencillez a partir de la formalización matemática de unas premisas condicionadas desde criterios y alternativas planteadas de manera previa a la realización del análisis (Arquero et al., 2009).

El AHP resulta aplicable a decisiones de tipo individual y grupal que pretendan una búsqueda de consenso entre los agentes implicados, puesto que a través de una construcción inteligible ofrece sencillez de implementación entre no matemáticos.

La aplicación de AHP se lleva a cabo mediante una comparación pareada de alternativas, dándole traslado a una matriz de comparaciones "par a par", tanto para criterios como para alternativas. Las matrices AHP deben garantizar su reciprocidad, robustez y consistencia (Saaty, 1980; Saaty, 1990).

La distribución de juicios y valores subyacentes muestra alta variabilidad entre individuos. De este modo, promediando de forma geométrica las opiniones de los cinco expertos participantes ${ }^{2}$, dotamos de universalidad y objetividad la propuesta de alternativas elegidas para los ejes ACEC (analizar, conectar, experimentar y crear).

Para la elaboración del AHP se tomaron en consideración la totalidad de competencias ${ }^{3}$ establecidas por la guía docente de la asignatura, resultando dos competencias generales y una competencia específica que distribuimos en dos subcompetencias por su redacción formal desde

2 El cuestionario remitido a cada uno de los expertos empleó la Escala de comparación Saaty (Saaty, 1980; Saaty, 1990) que establece un continuo de 9 a 1 para la primera opción comparada y de 1 a 9 para la segunda opción comparada en ese par, siendo 1 plena indiferencia entre ambas alternativas disponibles y los valores $3,5,7$ y 9 , moderada, fuerte, muy fuerte y extrema preferencia por el valor declarado. A estos valores los denominamos principales. Los valores 2, 4, 6 y 8 resultan posiciones centrales entre dos opciones, tales como 3 y 5,5 y 7 o 7 y 9 . A los valores 2, 4, 6 y 8 los denominaremos secundarios.

${ }^{3}$ CG7: Capacidad crítica y analítica en el área de especialidad de las Ciencias Sociales y Jurídicas; CG13: Capacidad de consolidación, ampliación e integración de los conocimientos fundamentales de la rama de Ciencias Sociales y Jurídicas; CE30A: Capacidad y habilidad para analizar la evolución del entorno económico y las características económicas del sector de empresas de la comunicación; CE30B: Capacidad y habilidad para tomar decisiones que se adapten a la evolución del entorno económico y a las características económicas del sector de empresas de la comunicación. la Universidad Miguel Hernández de Elche.

El AHP permite llevar a cabo una clasificación objetivada tanto de los criterios de análisis como de las alternativas disponibles a partir de su formulación y previa consulta de expertos en la materia objeto de decisión, diseñando un árbol de jerarquías a partir de la información procedente del trabajo matemático de normalización de matrices (anexos 3 y 4), que ofrece información precisa sobre el objetivo a alcanzar (en nuestro caso, propuesta de acción docente a realizar), las alternativas disponibles y los criterios aplicables en el contexto de la decisión 4 .

\section{Implementación en el aula}

Teniendo en cuenta la guía docente, se tomaron en consideración las actividades con mayor puntuación según el AHP, una para cada eje ACEC.

A continuación, se explican en profundidad las opciones elegidas que fueron implementadas en el aula:

AN1 - Lecturas propuestas por el profesor y exposición en clase de las conclusiones

Empleando Moodle los alumnos pudieron abordar una selección de textos de autores que analizan la transformación del sector digital desde las dimensiones social, política y económica.

A partir de estas lecturas se llevaron a cabo diversos debates utilizando las TIC. Por un lado, a través de un foro de debate en Moodle. Por otro, una serie de microdebates en Twitter empleando para ello el hashtag \#ComunicArte16.

Se eligió Twitter en base a numerosas investigaciones que lo consideran una herramienta apreciada por los profesionales de la comunicación porque permite interactuar con un formato breve, rápido y adecuado para lanzar titulares (Carrera et al., 2012), obligando a la síntesis en el mensaje. Twitter permite entablar conversaciones con los usuarios, contrastar opiniones y debatir puntos de vista (Watson, 2015). Dado que este trabajo se ha llevado a cabo en el grado en Comunicación y Relaciones Públicas, se ha considerado que Twitter es la red social que mejor se adapta a los requerimientos del estudio. Del mismo modo, se empleó Periscope, aplicación para la retransmisión de vídeo en streaming que se asocia a la cuenta de Twitter.

Los alumnos pudieron aprehender el modo en que el contexto 2.0 afecta a los agentes económicos involucrados en el sector de la comunicación.

CO2 - Visitas de expertos profesionales del sector de la comunicación al aula

Primera visita

Se invitó a los creadores de un medio de comunicación cuya particularidad se basa en su distribución gratuita a través de tablets, aprovechando todas sus posibilidades para servir contenidos interactivos diseñados expresamente. Esta sesión se realizó a través de un webinar en el que los alumnos tuvieron posibilidad de interactuar con los fundadores del medio.

El objeto de la sesión pasó por aproximar a los alumnos la vanguardia de medios digitales, y en modelos de negocio cuya monetización se sustenta sobre la publicidad.

Segunda visita

Un reconocido jurista ofreció una sesión en la que los

\footnotetext{
${ }^{4}$ El árbol de jerarquías desarrollado consta de cuatro ramas diferenciadas, coincidentes con cada una de las etapas para las cuales los alumnos previamente habían generado ideas aplicables al aula en modo de experiencias docentes, siguiendo la dinámica participativa expuesta anteriormente.
} 
alumnos pudieron conocer las implicaciones de la protección de derechos de imagen o la normativa en materia de protección de datos que le resulta afecta a la comunicación digital. Los alumnos tomaron conciencia de las relaciones legales del régimen de concursos en redes sociales como Facebook. Esta sesión y la siguiente fueron retransmitidas a través de Periscope.

Tercera visita

Un reputado profesional del ámbito de la consultoría estratégica y la innovación ofreció una interesante sesión sobre metodologías lean startup para la generación de nuevos modelos de negocio innovadores y disruptivos en el contexto del sector de la comunicación. Los alumnos pudieron tomar nota de los requerimientos propios del lanzamiento de una empresa de base tecnológica del sector de comunicación.

EX3 - Realizar una campaña publicitaria y difundirla en redes sociales, desarrollando un meme que pudiera ser empleado por una empresa real

Los alumnos eligieron una marca existente, tradicional o de base online, y sobre ella, emularon la creación de un meme de carácter no casual cuya finalidad era, con su difusión, lograr la mayor viralidad posible en redes sociales, afectando decisivamente a la notoriedad y al ROI de la campaña.

Estas campañas fueron secuenciadas y programadas a través de un cronograma por los alumnos, si bien no fueron difundidas en redes sociales, fundamentalmente, por no contar con la correspondiente autorización de dichas marcas.

CR2 - Crear un medio de comunicación y establecer cómo será su monetización

Aprovechando el trabajo reflexivo realizado en el eje analizar, los aprendizajes de tipo híbrido (innovadoremprendedor) del eje conectar y el expertise asimilado a lo largo de la etapa experimentar, los alumnos eligieron un target, configuraron una oferta a través de un medio de comunicación de nueva planta y diseñaron el modelo de negocio, su forma de generación de ingresos (vía publicidad, suscripción, mixto u otros) y su canal de distribución (online, mobile, etc.).

Del mismo modo, fijaron el marco de los canales de comunicación por los que actuaría, aproximando un presupuesto para dicho lanzamiento junto a la previsión orientativa de proyecciones financieras vía ingresos, estableciendo la fecha estimada del break even point.

\section{Análisis y valoración de ComunicArte2016}

Se suministró un cuestionario de satisfacción y se atendieron individualmente todo tipo de ideas y sugerencias relacionadas con la dinámica vivenciada por los alumnos, ofreciendo información cualitativa basada en la observación participante por parte del profesorado, de modo que resultó posible la obtención de información relevante y flexibilidad de interpretación (Blasco et al., 2016).

\subsubsection{Instrumentos de medición para la recogida de resultados}

Se preparó un cuestionario en escala Likert (1-5) que permitiera medir la aceptación de ComunicArte16 por parte del alumnado y su satisfacción tras haber diseñado las acciones docentes a realizar en el aula, obteniendo 14 resultados válidos de alumnos de entre 19 y 25 años, con una edad media de 20 años y 35\% hombres y el $65 \%$ mujeres (anexo 5). La tasa de respuesta del cuestionario representó un $73,68 \%$ del alumnado participante en el proyecto.
En el ámbito de la difusión en Twitter, la información recogida se obtuvo por medio de un recuento manual de tuits publicados con el hashtag \#ComunicArte16.

La participación activa del alumnado se analizó a partir de una metodología de tipo cualitativo, puesto que su visión panorámica de la realidad aporta riqueza y profundidad de análisis (LeCompte y Preissle, 1993).

Además, a fin de valorar el compromiso del alumnado, se tuvo en consideración el grado de absentismo registrado en la asistencia a clase.

\section{Resultados}

El análisis cuantitativo del cuestionario facilitado señala que dentro del ámbito de satisfacción del alumnado con el proyecto destaca el interés por la participación en ComunicArte16, con una media de 4,643, y en el lado de los resultados de aprendizaje destacan las percepciones favorables del alumnado sobre su capacidad de coordinación entre teoría y práctica $(4,429)$, así como la colaboración grupal llevada a cabo $(4,357)$. Sin embargo, pese a que la puntuación media no es destacadamente baja $(3,929)$, el interés del alumnado por la materia al haber participado en el diseño de las acciones docentes muestra una fuerte dispersión de resultados, apuntando en una cierta polarización de opiniones dentro del aula.

\begin{tabular}{|c|c|c|c|c|}
\hline & Minimo & Máximo & Media & $\begin{array}{c}\text { Desv. } \\
\text { Tip. }\end{array}$ \\
\hline \multicolumn{5}{|l|}{ Satisfacción con el proyecto } \\
\hline 1. Me suscitaba interés participar en ComunicArte 16 & 3 & 5 & 4,643 & 0,633 \\
\hline $\begin{array}{l}\text { 2. Mi interés hacia las experiencias docentes se ha } \\
\text { incrementado después de haber participado de forma activa en } \\
\text { su diseño y desarrollo }\end{array}$ & 3 & 5 & 4,286 & 0,611 \\
\hline $\begin{array}{l}\text { 3. Globalmente, estoy satisfecho de haber realizado este } \\
\text { proceso de diseño y desarrollo de experiencias docentes }\end{array}$ & 3 & 5 & 3,927 & 0,730 \\
\hline $\begin{array}{l}\text { 4. Me gustaría que se realizaran más procesos de diseño y } \\
\text { desarrollo de experiencias docentes en otras asignaturas de mi } \\
\text { carrera }\end{array}$ & 3 & 5 & 4,071 & 0,616 \\
\hline $\begin{array}{l}\text { 5. El profesor responsable nos ha integrado correctamente en } \\
\text { la dinámica de diseño y desarrollo de experiencias docentes }\end{array}$ & 3 & 5 & 4,143 & 0,770 \\
\hline $\begin{array}{l}\text { 6. El proceso de diseño y desarrollo de experiencias docentes } \\
\text { me ha parecido intelectualmente estimulante }\end{array}$ & 3 & 5 & 4,143 & 0,663 \\
\hline \multicolumn{5}{|l|}{ Resultados de aprendizaje } \\
\hline $\begin{array}{l}\text { 7. Mi interés por la materia estudiada en la asignatura ha } \\
\text { aumentado como consecuencia de la participación en el } \\
\text { proceso de diseño y desarrollo de experiencias docentes }\end{array}$ & 2 & 5 & 3,929 & 0,917 \\
\hline $\begin{array}{l}\text { 8. Al haber podido ser co-responsable de mi propio proceso de } \\
\text { aprendizaje, he conseguido una mayor coordinación entre la } \\
\text { teoría y práctica }\end{array}$ & 3 & 5 & 4,429 & 0,646 \\
\hline $\begin{array}{l}\text { 9. En conjunto, he mejorado la colaboración con otros } \\
\text { compañeros y con el profesor a través del proceso de diseño y } \\
\text { desarrollo de experiencias docentes }\end{array}$ & 3 & 5 & 4,357 & 0,633 \\
\hline $\begin{array}{l}\text { 10. Recomendaria a mis compañeros llevar a cabo un proceso } \\
\text { de diseño y desarrollo de experiencias docentes con las } \\
\text { caracteristicas de la que hemos abordado a lo largo del curso }\end{array}$ & 3 & 5 & 4,143 & 0,535 \\
\hline
\end{tabular}

Tabla 2. Resultados del cuestionario de satisfacción con el proyecto y de valoración de los resultados de aprendizaje. Fuente: Elaboración propia

En la difusión en Twitter y Periscope de las diferentes acciones que configuraron ComunicArte16 participó de forma activa en torno a un $40 \%$ del alumnado. Se publicaron 33 tuits durante 11 semanas, logrando un total de 19.548 impresiones, 894 interacciones y una tasa de interacción media del 4,72\%. Los tuits correspondientes con la emisión en Periscope de las visitas de expertos acumularon 299 interacciones alcanzando un pico del 10,25\% en la tasa de interacción (anexo 6).

Tanto en las sesiones grupales como en las tutorías, así como en el registro de interacciones a través del entorno virtual Moodle, se constató un incremento gradual de la participación activa de los alumnos a lo largo del curso. Las dinámicas grupales de generación de propuestas y la 
Castelló-Sirvent, Fernando y Roger Monzó, Vanessa (2018). El papel del alumnado en la propuesta y diseño de acciones docentes. @tic revista d'innovació educativa, 20, 1-15.

receptividad en la puesta en funcionamiento de las diferentes acciones docentes previamente diseñadas mostraron avances en el nivel de implicación del alumnado en el seguimiento de la asignatura, fundamentalmente por su fuerte vinculación al reconocerse como protagonistas de su propio aprendizaje.

El compromiso del alumnado mostró una significativa mejora al registrar una reducción en la tasa de faltas de asistencia de un $8,75 \%$ con respecto al curso inmediatamente anterior en el que no se aplicó este proyecto.

\section{Conclusiones}

Fomentar la originalidad de los alumnos resulta un ámbito competencial que se presenta como central para garantizar su empleabilidad en el mercado laboral.

Son múltiples los trabajos que han aportado mecanismos de detección de los diferentes estilos de aprendizaje FSLSM preferidos por el alumnado para lograr mejoras en su implicación y rendimiento.

Por otro lado, un adecuado ajuste de los estilos de enseñanza con respecto a los estilos de aprendizaje representa uno de los elementos propios del diseño ACEC, en tanto que modelo integrador. En este sentido, comprobamos que ComunicArte16 fue muy bien valorado por el alumnado participante. El análisis del proyecto descrito en este trabajo devuelve una amplia mejora en la satisfacción del alumnado, al tiempo que estimula su proactividad e impacta positivamente sobre sus niveles de participación y compromiso a lo largo del curso.

El uso de dinámicas de grupo basadas en soporte TIC puede lograr una mayor y mejor vinculación del alumnado con su propio currículo puesto que les ofrece la posibilidad de situarse en el centro de su aprendizaje. La participación del alumnado en el diseño de acciones docentes mejora la motivación y ofrece al profesorado alta retroalimentación por parte del alumno, por cuanto indica, sugiere y propone aquellas iniciativas que entiende más adecuadas desde su punto de vista, hecho que, en definitiva, le vuelve más permeable a lo largo del proceso de aprendizaje.

Por otra parte, diversos autores (Castaño et al., 2015; Paulsen y Feldman, 2015; Danielsen et al., 2009) han destacado la influencia existente entre satisfacción y rendimiento. Sin embargo, por la metodología empleada, este trabajo encuentra una limitación en la ausencia de evidencia respecto a este correlato.

Futuras líneas de investigación deben orientar su acción a integrar la detección temprana de estilos de aprendizaje FSLSM, a partir de la huella digital de los alumnos en los entornos virtuales de aprendizaje (LMS) y validar estadísticamente la consistencia del Modelo ACEC en términos de mejora del rendimiento académico, tanto en evaluación continua como en examen final. La ampliación del número y variedad de redes sociales aplicadas en un enfoque bidireccional (alumno-profesor y profesor-alumno), junto al análisis de la autoevaluación de los resultados de aprendizaje y su comparación por diferencias de medias, puede contribuir a un mejor conocimiento de las relaciones subyacentes.

\section{Bibliografía}

Arancibia-Herrera, M., Oliva-Figueroa, I. y Paiva-Cornejo, F. (2014). Procesos de significación mediados por una plataforma de aprendizaje colaborativo desde los protagonistas. Comunicar: Revista Científica de Comunicación y Educación, 21(42), 75-85.DOI: http://dx.doi.org/10.3916/C42-2014-07

Arquero, A., Álvarez, M. y Martínez, E. (2009).Decision management making by AHP (Analytical Hierarchy Process) through GIS data. IEEE Latin America Transactions, 7(1), 101-106. DOI: http://dx.doi.org/10.1109/TLA.2009.5173471

Ballesta, F., Izquierdo, T. y Romero, B. (2011). Percepción del alumnado de Pedagogía ante el uso de metodologías activas. Educatio Siglo XXI, 29(2), 353-368. Recuperado de: http://revistas.um.es/educatio/article/view/133101

Baños, R., del Mar Ortiz-Camacho, M.; Baena-Extremera, A. y Tristán-Rodríguez, J. L. (2017). Satisfacción, motivación y rendimiento académico en estudiantes de Secundaria y Bachillerato: antecedentes, diseño, metodología y propuesta de análisis para un trabajo de investigación. Espiral. Cuadernos del profesorado, 10(20), 40-50. DOI: http://dx.doi.org/10.25115/ecp.v10i20.1011

Barajas Meneses, F. y Álvarez Morán, C. (2013). Uso de Facebook como herramienta en la enseñanza del área de naturales en el grado undécimo de educación media vocacional. Píxel-Bit: Revista de medios y educación, 42, 143-156. Recuperado de: http://ir.uv.es/G1mYNv8

Bernal-Bravo, C., y Angulo-Rasco, F. (2013). Interacciones de los jóvenes andaluces en las redes sociales. Comunicar: Revista Científica de Comunicación y Educación, 20(40), 25-30. DOI: http://dx.doi.org/10.3916/C40-2013-02-02

Berumen, S. A. y Llamazares, F. (2007). La utilidad de los métodos de decisión de muestreo (como el AHP) en un entorno de competitividad creciente. Cuadernos de Administración, 20(34), 65-87. Recuperado de: http://revistas.javeriana.edu.co/index.php/cuaderno s_admon/article/view/4043

Blasco, A. C., Guerrero Martínez, J. F. y Sarsa Garrido, J. (2016). La clase invertida y el uso de vídeos de software educativo en la formación inicial del profesorado. Estudio cualitativo. @tic revista d'innovació educativa, 17, 12-20. DOI: http://doi.org/10.7203/attic.17.9027

Cabero Almenara, J. y Marín Díaz, V. (2014). Posibilidades educativas de las redes sociales y el trabajo en grupo. Percepciones de los alumnos universitarios. Comunicar: Revista Científica de Comunicación y Educación, 21(42), 165-172. DOI: http://dx.doi.org/10.3916/C42-2014-16.

Cabero Almenara, J., Morales Lozano, J. A., Sánchez, F. M., Ballesteros-Regaña, C., Tena, R. R., Osuna, J. B. y de la Serna, M. C. (2006). Formación del profesorado universitario en estrategias metodológicas para la incorporación del aprendizaje en red en el Espacio Europeo de Educación Superior (EEES). Píxel-Bit: Revista de medios y educación, 27, 11-29. Recuperado de: https://recyt.fecyt.es/index.php/pixel/article/view/6 $1267 / 37281$

Carrera, P., Sainz de Baranda, C., Herrero, E., y Limón, N. (2012). Journalism and Social Media: How Spanish 
Castelló-Sirvent, Fernando y Roger Monzó, Vanessa (2018). El papel del alumnado en la propuesta y diseño de acciones docentes. @tic revista d'innovació educativa, 20, 1-15.

Journalists Are Using Twitter. Estudios sobre el Mensaje Periodístico, 18(1), 31-53. DOI: http://dx.doi.org/10.5209/rev_eSMP.2012.v18.n1.3 9353

Castaño, C., Maíz, I. y Garay, U. (2015). Diseño, motivación y rendimiento en un curso MOOC cooperativo. Comunicar: Revista Científica de Comunicación y Educación, 22(44), 19-26. DOI: https://doi.org/10.3916/C44-2015-02

Chang, Y. H., Chen, Y. Y., Chen, N. S., Lu, Y. Te y Fang, R. J. (2016). Yet another adaptive learning management system based on Felder and Silverman's Learning Styles and Mashup. Eurasia Journal of Mathematics, Science and Technology Education, 12(5), 12731285.

https://doi.org/10.12973/eurasia.2016.1512a

Danielsen, A. G., Samdal, O., Hetland, J., y Wold, B. (2009). School-related social support and students' perceived life satisfaction. Journal of Education Research, 102(4), 303-318. DOI: https://doi.org/10.3200/JOER.102.4.303-320

DeTombe, D. J. (2001). Methodology for Handling Complex Societal Problems. European Journal of Operational Research, 128(2), 227-230. DOI: http://dx.doi.org/10.1177/075910630006800121

Domingo, M. y Fuentes, M. (2010). Innovación educativa: experimentar con las TIC y reflexionar sobre su uso. Píxel-Bit. Revista de Medios y Educación, 36, 171180. Recuperado de: http://ir.uv.es/xOwvxoB

Felder, R. M. y Brent, R. (2001). Effective strategies for cooperative learning. Journal of Cooperation \& Collaboration in College Teaching, 10(2), 69-75. DOI: https://doi.org/10.1016/j.sbspro.2014.01.890

Felder, R. y Silverman, L. (1988). Learning and teaching styles in engineering education. Engineering Education, 78(7), 674-681. Recuperado de: http://www4.ncsu.edu/unity/lockers/users/f/felder/ public/Papers/LS-1988.pdf

Fu, G. S. y Li, Y. F. (2014). Group differences: E-learning anxiety and sense of spatial orientation - Groups divided by Felder-Silverman Learning Style. Proceedings - 2014 International Conference of Educational Innovation Through Technology, EITT 2014, 176-183. https://doi.org/10.1109/EITT.2014.36

García Pérez, F. F. (2000). Los modelos didácticos como instrumento de análisis y de intervención en la realidad educativa. Biblio $3 W$. Revista Bibliográfica de Geografía y Ciencias Sociales, 207, 1-10. Recuperado de: http://www.ub.edu/geocrit/b3w207.htm

Goikoetxea, E. y Pascual, G. (2005). Aprendizaje Cooperativo: Bases teóricas y hallazgos empíricos que explican su eficacia. Educación XX1, 5, 227247. DOI: https://doi.org/10.5944/educxx1.5.1.392

Gómez-Camarero, C., Palomares-Perraut, R. y Pino-Díaz, J. (2010). La utilización de herramientas colaborativas 2.0 en el ámbito de la documentación publicitaria. DIM: Didáctica, innovación y multimedia, 6(18). Recuperado de: https://www.raco.cat/index.php/dim/article/viewFile /214699/284991
González, M. (2016). Formación docente en competencias tic para la mediación de aprendizajes en el proyecto canaima educativo // Teacher training in ICT skills in the mediation of learning in the Canaima Educational Project. Telos, 18(3), 492-507. Recuperado https://trove.nla.gov.au/version/246734731

González-Peiteado, M. y Aznar-Cuadrado, V. (2010). Aproximación al perfil de estilos de enseñanza en la formación inicial del profesorado de Educación Secundaria: nuevos canales, nuevos retos. Actas del I Congreso Internacional Reinventar la profesión docente. Málaga: Universidad de Málaga, 348-356.

González-Peiteado, M. y Pino-Juste, M. (2013). Percepción del alumnado de Ciencias de la Educación de la Universidad de Santiago de Compostela sobre el uso de los estilos de enseñanza. Innovación Educativa, 23, 215-229. Recuperado de: http://www.usc.es/revistas/index.php/ie/article/vie wFile/782/1512

Gras Martí, A., y Cano Villalba, M. (2005). Debates y tutorías como herramientas de aprendizaje para alumnos de ciencia: análisis de la integración curricular de recursos del campus virtual. Enseñanza de las Ciencias, 23(2), 167-180. Recuperado de: https://www.raco.cat/index.php/ensenanza/article/v iew/22016

Guitert, M. y Pérez-Mateo, M. (2013). La colaboración en la red: hacia una definición de aprendizaje colaborativo en entornos virtuales. Teoría de la Educación 14(1), 10-31. http://ir.uv.es/2Wzs2Ht

Jingyun, W. y Takahiko, M. (2015). The Reliability and Validity of Felder-Silverman Index of Learning Styles in Mandarin Version. Information Engineering Express, 1(3), 1-8. Recuperado de: http://www.iaiai.org/journals/index.php/IEE/article/v iewFile/38/32

Joseph, L. y Abraham, S. (2017). Instructional design for learning path identification in an e-learning environment using felder-silverman learning styles model. 2017 International Conference on Networks \& Advances in Computational Technologies (NetACT), 215-220.

DOI: https://doi.org/10.1109/NETACT.2017.8076769

LeCompte, M. y Preissle, J. (1993). Ethnography and Qualitative Design in Educational Research. London: Academic Press.

Martín, A., León, C. y García, A. (2014). Innovación docente para la integración de autoformación y autoevaluación en la plataforma web. Píxel-Bit. Revista de Medios y Educación, 44, 201-214. DOI: http://dx.doi.org/10.12795/pixelbit.2014.i44.14

Martínez de Miguel, S. (2007). Una experiencia de innovación del portafolio del alumno en la diplomatura de educación social, desde el marco de la educación superior en Europa. Educatio Siglo XXI, 25, 125-144. Recuperado de: http://revistas.um.es/educatio/article/view/724

Ministerio de Educación, Cultura y Deportes (2003). La integración del Sistema Universitario Español en el Espacio Europeo de Enseñanza Superior. Documento Marco. Madrid: MECD. Recuperado de: 
Castelló-Sirvent, Fernando y Roger Monzó, Vanessa (2018). El papel del alumnado en la propuesta y diseño de acciones docentes. @tic revista d'innovació educativa, 20, 1-15.

http://www.ub.edu/comint/pdi/docs/docmarcmecd. pdf

Monereo, C. y Pozo, J.I. (2003). La universidad ante la nueva cultura educativa. Madrid: Síntesis.

Muruganandam, S. y Srinivasan, N. (2016). Appraisal of Felder-Silverman Learning Style Model with Discrete Data Sets. Indian Journal of Science and Technology (9).

DOI: http://dx.doi.org/10.17485/ijst/2016/v9i10/88992

Nonaka, I. y Takeuchi, H. (1995). The knowledge-creating company: How Japanese companies create the dynamics of innovation. Oxford: Oxford University Press.

Olmedo, E. M. (2013). Enfoques de aprendizaje de los estudiantes y metodología docente: Evolución hacia el nuevo sistema de formación e interacción propuesta en el EEES. Revista de investigación educativa, RIE, 31(2), 411-429. DOI: http://dx.doi.org/10.6018/rie.31.2.133501

Olmos-Migueláñez, S., Martínez-Abad, F., TorrecillaSánchez, E. M. y Mena-Marcos, J. J. (2014). Análisis psicométrico de una escala de percepción sobre la utilidad de Moodle en la universidad. RELIEVE, 20(2) DOI: https://doi.org/10.7203/relieve.20.2.4221

Özyurt, Ö., Özyurt, H., Baki, A. y Güven, B. (2013). Integration into mathematics classrooms of an adaptive and intelligent individualized e-learning environment: Implementation and evaluation of UZWEBMAT. Computers in Human Behavior, 29(3), 726-738.

https://doi.org/10.1016/j.chb.2012.11.013

Osborn, A. F. (1963). Applied Imagination; Principles and Procedures of Creative Problem-solving: Principles and Procedures of Creative Problem-solving. Scribner.

Paulsen, M. B. y Feldman, K. A. (2005). The conditional and interaction effects of epistemological beliefs on the self-regulated learning of college students: Motivational strategies research in higher education. Research in Higher Education, 46, 731-768. DOI https://doi.org/10.1007/s11162-004-6224-8

Piscitelli, A., Adaime, I., y Binder, I. (2010). El proyecto Facebook y la posuniversidad. Madrid: Ariel.

Porlán, R. (1993). Constructivismo y escuela. Hacia un modelo de enseñanza-aprendizaje basado en la investigación. Sevilla: Díada Editora.

Qodad, A., Tetouan, E., Yadari, M. E. L. y Kenz, A. El. (2016). An Adaptive Learning System based on a Job Model, the Differentiated Instruction and Felder and Silverman's Learning Styles Model. Information Science and Technology (CiSt), 2016 4th IEEE International Colloquium, 506-510. DOI: http://dx.doi.org/10.1109/CIST.2016.7805100

Roger-Monzó, V., Guijarro-García, M. y Martí-Sánchez, M. (2015). Digital Signage: An Experience of Innovation in Higher Education. Multidisciplinary Journal for
Education, Social and Technological Sciences, 2(2), 1-13. http://dx.doi.org/10.4995/muse.2015.3769

Ramírez León, Y. del V., y Rosas Espín, D. (2014). Aplicación de la teoría de estilos de aprendizaje al diseño de contenidos didácticos en entornos virtuales. Etic@net, 2 (14), 176-197. Recuperado de: http://ir.uv.es/uGoBzK6

Saaty, T. L. (1980). The analytic hierarchy process: planning, priority setting, resources allocation. New York, NY: McGraw.

Saaty, T. L. (1990). How to make a decision: the analytic hierarchy process. European journal of operational research, 48(1), 9-26.

Tejada, J. (2012). La alternancia de contextos para la adquisición de competencias profesionales en escenarios complementarios de educación superior: marco y estrategia. Educación XX1, 15 (2), 17-40. DOI: https://doi.org/10.5944/educxx1.15.2.125

Torregrosa, A. (2010). Uso de las TIC: Las redes sociales en el contexto educativo. Revista Funcae Digital, 12, 390-396. Recuperado de: http://ir.uv.es/i92UbI9

Truong, H. M. (2016). Integrating learning styles and adaptive e-learning system: Current developments, problems and opportunities. Computers in Human Behavior, 55, 1185-1193. DOI: https://doi.org/10.1016/j.chb.2015.02.014

Vallet-Bellmunt, T.; Rivera-Torres, P.; Vallet-Bellmunt, I. y Vallet-Belmunt, A. (2017). Aprendizaje cooperativo, aprendizaje percibido y rendimiento académico de la enseñanza de marketing. Educación XX1, 20(1), 277297. DOI: https://doi.org/10.5944/educxx1.17512

Vélez Van, M.A., Roa, N.C. (2005). Factors associated with academic performance in medical students. PSIC. Educación Médica, 2(8), 1-10.

Ventura, A. C., Palou, I., Széliga, C. y Angelone, L. (2014). Estilos de Aprendizaje y Enseñanza en Ingeniería: Una propuesta de Educación Adaptativa para primer Año. Revista Educación en Ingeniería, 9(18), 178189. DOI: http://dx.doi.org/10.26507/rei.v9n18.461

Watson, B. R. (2015). Is Twitter an alternative medium? comparing gulf coast Twitter and newspaper coverage of the 2010 bp oil spill. Communication Research,. 43(5), 647-671. DOI: http://dx.doi.org/10.1177/009365021456589

\section{| Cita recomendada de este artículo}

Castelló-Sirvent, Fernando y Roger Monzó, Vanessa (2018). El papel del alumnado en la propuesta y diseño de acciones docentes. @tic revista d'innovació educativa, $20,1-15$. 
Castelló-Sirvent, Fernando y Roger Monzó, Vanessa (2018). El papel del alumnado en la propuesta y diseño de acciones docentes. @tic revista d'innovació educativa, 20, 1-15.

\section{A1. Estructura del Proyecto de Innovación ComunicArte16}

\begin{tabular}{|c|c|c|c|c|}
\hline \multirow[t]{2}{*}{ Programación secuencial de las fases } & \multirow[t]{2}{*}{ Dedicación } & \multicolumn{3}{|c|}{ Participantes involucrados } \\
\hline & & Profesores & Alumnos & Expertos \\
\hline $\begin{array}{l}\text { 1. Presentación inicial } \\
\begin{array}{l}\text { - } \\
\text { - } \\
\text { - } \\
\text { - ACEmunignatura } \\
\end{array}\end{array}$ & 2 horas & $\begin{array}{l}X \\
X \\
X\end{array}$ & $\begin{array}{l}X \\
X \\
X\end{array}$ & \\
\hline $\begin{array}{l}\text { 2. Generación de propuestas } \\
\begin{array}{l}\text { - } \\
\text { - } \text { Brainstorming } \\
\text { Debates }\end{array}\end{array}$ & 10 horas & $\begin{array}{l}X \\
X\end{array}$ & $\begin{array}{l}X \\
X\end{array}$ & \\
\hline $\begin{array}{l}\text { 3. Selección de alternativas } \\
\begin{array}{l}\text { - } \\
\text { - } \\
\text { - } \\
\text { - } \\
\text { Selelusión en bación de preferencias de opciones (AHP) }\end{array}\end{array}$ & $(* *)$ & $\begin{array}{l}x \\
x\end{array}$ & & $\mathrm{x}$ \\
\hline $\begin{array}{l}\text { 4. Implementación en el aula } \\
\begin{array}{l}\text { - Experiencia Etapa de Analizar } \\
\text { - Experiencia Etapa de Conectar } \\
\text { - Experiencia Etapa de Experimentar } \\
\text { - Experiencia Etapa de Crear }\end{array}\end{array}$ & 16 horas & $\begin{array}{l}X \\
X \\
X \\
X\end{array}$ & $\begin{array}{l}X \\
X \\
X \\
X\end{array}$ & \\
\hline $\begin{array}{l}\text { 5. Análisis y evaluación de ComunicArte2016 } \\
\text { - Cuestionario de satisfacción } \\
\text { - Atención de sugerencias }\end{array}$ & 2 horas & $\begin{array}{l}X \\
X\end{array}$ & $\begin{array}{l}X \\
X\end{array}$ & \\
\hline Fase de docencia contenidos teóricos (*) & 30 horas & $x$ & $x$ & \\
\hline
\end{tabular}

${ }^{*}$ ) Discurre en paralelo a las fases 2,3 y 4

$(* *)$ No aplica, puesto que se celebra fuera del horario lectivo

Fuente: Elaboración propia 
Castelló-Sirvent, Fernando y Roger Monzó, Vanessa (2018). El papel del alumnado en la propuesta y diseño de acciones docentes. @tic revista d'innovació educativa, 20, 1-15.

\section{A2. Selección del profesorado de las acciones docentes iniciales propuestas por el alumnado}

\begin{tabular}{l}
\hline Analizar \\
\hline AN1 - Lecturas propuestas por el profesor y exposición en clase de las conclusiones. \\
AN2 - Trabajos en grupo consultando materiales de Internet y de biblioteca sobre la organización económica \\
de empresas del sector de la comunicación. \\
AN3 - Debates en el aula a partir del análisis de lecturas recomendadas por el profesor. \\
AN4 - Realizar encuestas a diferentes empresas del sector de la comunicación. \\
\hline Conectar \\
\hline CO1 - Visionado de películas y series de temática periodística. \\
CO2 - Visitas de expertos profesionales del sector de la comunicación al aula. \\
CO3 - Visitas a agencias y medios de comunicación. \\
CO4 - Asistencia a conferencias relacionadas con la temática de la asignatura. \\
Experimentar \\
EX1 - Crear un hashtag en Twitter y utilizar técnicas de social media que permitan convertirlo en TT local. \\
EX2 - Iniciar un rumor en Facebook difundiéndolo en el muro de cada uno de los estudiantes. \\
EX3 - Realizar una campaña publicitaria y difundirla en redes sociales, desarrollando un meme que pudiera \\
ser empleado por una empresa real. \\
\hline Crear \\
\hline CR1 - Desarrollar la estructura organizativa y económica de un medio de comunicación ya existente. \\
CR2 - Crear un medio de comunicación y establecer cómo será su monetización. \\
CR3 - Crear una campaña viral incidiendo especialmente en el soporte mobile.
\end{tabular}

Fuente: Elaboración propia

A3. Resultados del vector promedio del AHP para criterios de elección y de las opciones ganadoras

\begin{tabular}{lllll}
\hline & CG7 & CG13 & CE30A & CE30B \\
\hline Analizar & 0,33 & 0,31 & 0,27 & 0,06 \\
\hline Conectar & 0,30 & 0,29 & 0,29 & 0,09 \\
\hline Experimentar & 0,11 & 0,35 & 0,18 & 0,33 \\
\hline Crear & 0,10 & 0,44 & 0,11 & 0,33 \\
\hline CLASIFICACIÓN & Analizar & Conectar & Experimentar & Crear \\
\hline $1^{\text {a }}$ & AN1 $(0,35)$ & CO2 $(0,42)$ & EX3 $(0,43)$ & CR2 $(0,62)$ \\
\hline $2^{\text {a }}$ & AN2 $(0,30)$ & CO1 $(0,24)$ & EX2 $(0,39)$ & CR3 $(0,26)$ \\
\hline $3^{a}$ & AN3 $(0,25)$ & CO3 $(0,23)$ & EX1 $(0,15)$ & CR1 $(0,11)$ \\
\hline $4^{\text {a }}$ & AN4 $(0,10)$ & CO4 $(0,08)$ & & - \\
\hline
\end{tabular}

Fuente: Elaboración propia 
Castelló-Sirvent, Fernando y Roger Monzó, Vanessa (2018). El papel del alumnado en la propuesta y diseño de acciones docentes. @tic revista d'innovació educativa, 20, 1-15.

\section{A4. Árbol Jerárquico del AHP}

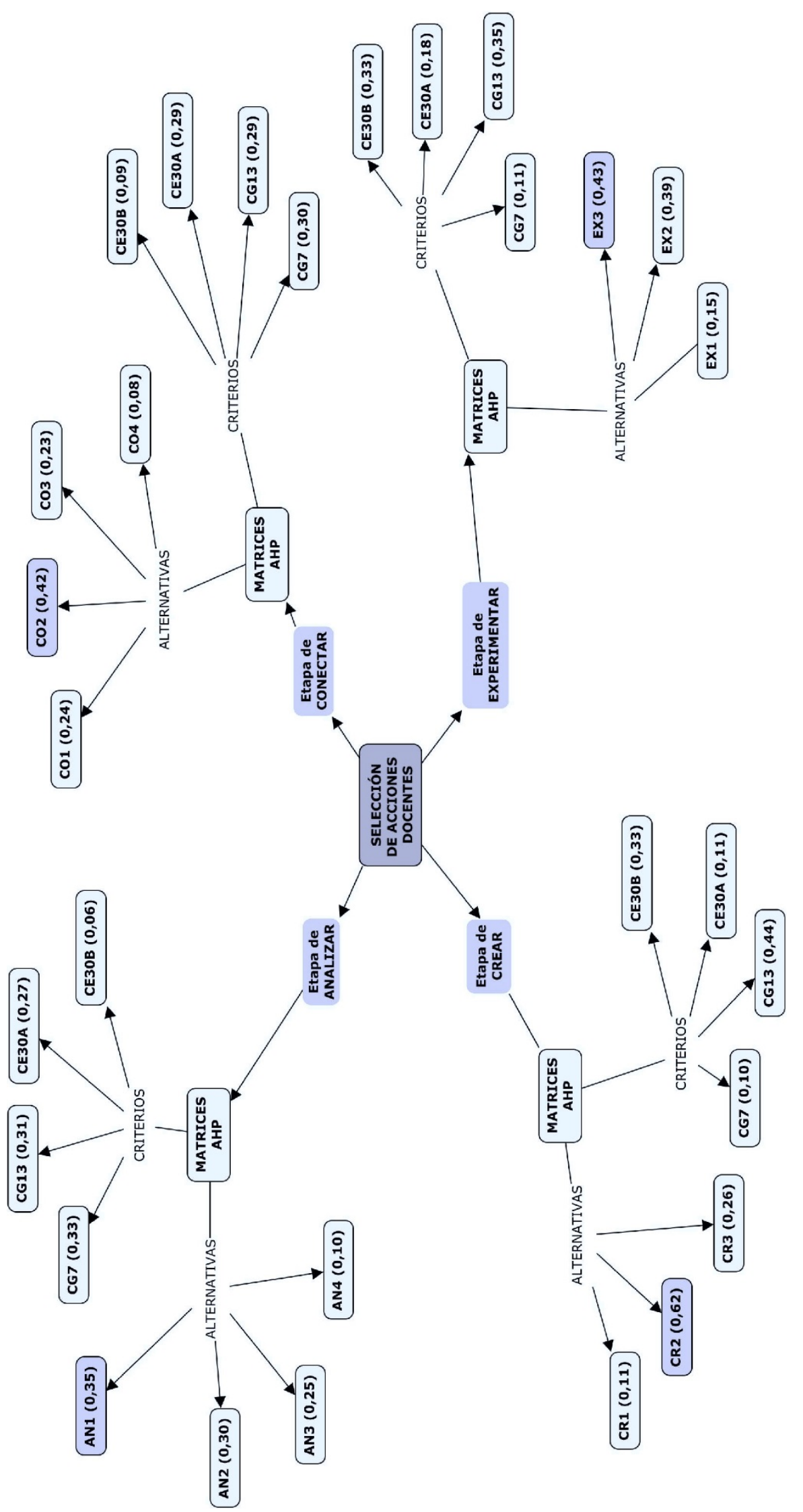

Fuente: Elaboración propia 
Castelló-Sirvent, Fernando y Roger Monzó, Vanessa (2018). El papel del alumnado en la propuesta y diseño de acciones docentes. @tic revista d'innovació educativa, 20, 1-15.

\section{A5. Ficha técnica de la investigación y perfil de los alumnos encuestados}

\begin{tabular}{|c|c|}
\hline Universo & $\begin{array}{l}\text { Estudiantes universitarios de la asignatura Estructura } \\
\text { Económica del Sector de la Comunicación }\end{array}$ \\
\hline Ámbito geográfico & Valencia, España \\
\hline Diseño muestral & Encuesta personal \\
\hline Tamaño de la muestra & 14 cuestionarios válidos \\
\hline Trabajo de campo & $3 / 5 / 2016$ \\
\hline Técnicas estadísticas & Análisis descriptivo \\
\hline \multirow[t]{2}{*}{ Software empleado } & SPSS versión 21.0 \\
\hline & Peso porcentual \\
\hline \multirow[t]{2}{*}{ Género } & Hombres \\
\hline & Mujeres \\
\hline Edad & $\begin{array}{lc}\text { Media } & 20,79 \\
\text { Desviación típica } & 1,89 \\
\text { Mínimo } & 19 \\
\text { Máximo } & 25\end{array}$ \\
\hline
\end{tabular}

Fuente: Elaboración propia 
Castelló-Sirvent, Fernando y Roger Monzó, Vanessa (2018). El papel del alumnado en la propuesta y diseño de acciones docentes. @tic revista d'innovació educativa, 20, 1-15.

\section{A6. Resultados de difusión en Twitter con el hashtag \#ComunicArte16}

\begin{tabular}{|c|c|c|c|c|}
\hline Fecha & Impresiones & Interacciones & Tasa & RT \\
\hline Total & 19.548 & 894 & $4,72 \%$ & 66 \\
\hline Media & 610,86 & 27,94 & $4,72 \%$ & \\
\hline $12 / 1 / 16$ & 1.427 & 125 & $8,76 \%$ & 8 \\
\hline $12 / 1 / 16$ & 770 & 26 & $3,38 \%$ & 3 \\
\hline $29 / 1 / 16$ & 199 & 2 & $1,01 \%$ & \\
\hline $1 / 2 / 16$ & 377 & 13 & $3,45 \%$ & 1 \\
\hline $3 / 2 / 16$ & 426 & 5 & $1,90 \%$ & \\
\hline $5 / 2 / 16$ & 335 & 10 & $2,99 \%$ & \\
\hline $16 / 2 / 16$ & 255 & 5 & $1,96 \%$ & 1 \\
\hline $16 / 2 / 16$ & 367 & 7 & $2,99 \%$ & \\
\hline $17 / 2 / 16$ & 189 & 2 & $1,06 \%$ & \\
\hline $23 / 2 / 16$ & 359 & 29 & $8,08 \%$ & \\
\hline $24 / 2 / 16$ & 232 & 1 & $0,43 \%$ & \\
\hline $26 / 2 / 16$ & 178 & 5 & $2,81 \%$ & \\
\hline $28 / 2 / 16$ & 217 & 3 & $1,38 \%$ & \\
\hline $3 / 3 / 16$ & 2.614 & 79 & $3,02 \%$ & 6 \\
\hline $3 / 3 / 16$ & 323 & 15 & $4,64 \%$ & \\
\hline $5 / 3 / 16$ & 367 & 3 & $1,24 \%$ & \\
\hline $6 / 3 / 16$ & 564 & 26 & $5,37 \%$ & 4 \\
\hline $12 / 3 / 16$ & 231 & 6 & $2,60 \%$ & \\
\hline $14 / 3 / 16$ & 256 & 2 & $1,01 \%$ & \\
\hline $26 / 3 / 16$ & 384 & 7 & $1,99 \%$ & 1 \\
\hline $29 / 3 / 16$ & 191 & 3 & $1,57 \%$ & \\
\hline $30 / 3 / 16$ & 193 & 3 & $1,55 \%$ & \\
\hline $7 / 4 / 16$ & 2.355 & 84 & $3,57 \%$ & 9 \\
\hline $8 / 4 / 16$ & 957 & 54 & $5,64 \%$ & 3 \\
\hline $8 / 4 / 16$ & 885 & 54 & $6,10 \%$ & 3 \\
\hline $22 / 4 / 16$ & 277 & 3 & $1,08 \%$ & 1 \\
\hline $22 / 4 / 16$ & 179 & 1 & $0,56 \%$ & \\
\hline $4 / 5 / 16$ & 336 & 3 & $0,89 \%$ & 1 \\
\hline $22 / 4 / 16$ & 1.054 & 108 & $10,25 \%$ & 7 \\
\hline $22 / 4 / 16$ & 1.500 & 137 & $9,13 \%$ & 12 \\
\hline 4/4/16 & 1.081 & 29 & $2,68 \%$ & 4 \\
\hline $12 / 4 / 16$ & 470 & 44 & $9,36 \%$ & 2 \\
\hline
\end{tabular}

Fuente: Elaboración propia 